\title{
Modeling and Identification for the Design of a Rotary Soft Actuator based on Wren Mechanism
}

\author{
Thibault Gayral ${ }^{1}$, Lennart Rubbert ${ }^{1}$ and Pierre Renaud ${ }^{1}$
}

\begin{abstract}
In this paper, we describe the use of calibration techniques to help improving prediction accuracy of models for soft actuators. A finite-difference model considering anisotropy of soft actuator flexible chamber is combined with experimental identification to assess actuator motion. Identifiability conditions are exploited to determine suitable experimental conditions, taking into account the manufacturing process capabilities, multimaterial additive manufacturing in this context. As a second contribution, a soft actuator with Wren mechanism for reinforcement is being developed. It is considered for rotary actuation, with the description of a proof of concept. The interest of the calibration technique and the actuator design are finally discussed.
\end{abstract}

\section{INTRODUCTION}

Soft actuators are of interest for soft robot design [1] and also to develop devices such as medical systems [2], for the safety their compliance can offer. Pneumatic soft actuators combine compliance and high power-to-volume ratio. Their number increases, notably with well-known pneumatic artificial muscles (PAM) [3], [4] based on fiber reinforcement of a flexible chamber, pleated structures [5], origami-based actuators [6], bellow-based flexible actuators [7]. In the latter, soft actuation was then combined to inchworm kinematics for the insertion of surgical tools with high level of safety.

All designs share the use of a flexible chamber under pressure, with an anisotropy that can be obtained by the chamber shape, the material distribution and the material anisotropy. In [2], we considered the use of two polymers of very different stiffness properties in order to build anisotropic cylindrical chamber. The design is then simple to implement using molding or more interestingly using multimaterial additive manufacturing (MMAM). MMAM was successfully considered for the design of soft actuator [8], and such process makes possible the production of functionally graded materials [9], with strong interfaces between the materials [10]. New designs of soft actuators should therefore emerge from the use of MMAM.

The capability to design new soft actuators is linked to the possibility to predict their performances. Starting from model of the materials and the deformation of flexible chambers, analytical models and finite element analysis (FEA) have been considered [11], [12], sometimes after an identification [13]. Representation models were also introduced in particular for

\footnotetext{
*This work was supported by the INTERREG Upper Rhine program from the ERDF (European Regional Development Fund), SPIRITS project, and also by Investissements d'Avenir program (Labex CAMI \& Equipex ROBOTEX)

${ }^{1}$ ICube laboratory, INSA - CNRS - University of Strasbourg thibault.gayraleinsa-strasbourg.fr
}

control [14]. If PAM kinematics are now well described [15], it is yet difficult to build accurate analytical kinematics models for any geometry of soft actuator. When the actuator length is short with respect to its diameter, pressure effect is indeed difficult to model [7] while such geometries are of interest for the design of gripper or inchworm-based actuators [7], [2]. FEA can be helpful but it is difficult, when MMAM is considered, to model the interactions between different materials, the impact of process resolution and variability as observed in [10]. In addition, FEA is delicate to use during design if the influence of all design parameters is not yet known.

In [16], models from continuum mechanics were interestingly used, considering the actuator composed of an anisotropic material. With MMAM, one can indeed see the production of an actuator chamber as the production of a composite material. As described above, this composite material is however difficult to model finely because of process uncertainties and complexity of material interactions at a microscopic level. One advantage of digital fabrication techniques is the possibility to produce easily and at low cost prototypes. In this paper, we therefore investigate the use of calibration techniques to refine model. Our proposition is twofold. We consider a model of intermediate complexity, initially developed in [17], [18] to capture the complexity of deformations of small flexible chambers. In addition, we integrate identification techniques to refine the model, using identifiability conditions developed in [19] in order to ensure a correct identification of model in presence of process and experiment uncertainties.

This paper is focused on the introduction of a calibrationbased technique for modeling of soft actuator. As the second contribution, we use this method to develop a new flexible actuator. The actuator is composed of a flexible chamber with reinforcement by means of a compliant Wren mechanism [20], which interest will be justified in the next section. Our final goal is the development of a robotic component for the medical field. The case study is the development of a needle driver with control of needle rotation around its axis by means of a rotary soft actuator, of use in interventional radiology [2], [7], [21].

In section II, the Wren mechanism properties are outlined to justify its interest for soft actuator design. The modeling of soft actuator is then derived in section III. Section IV is dedicated to calibration. A proof of concept of rotary actuator is presented in section $\mathrm{V}$ with discussion on the interest of the calibration process. Conclusions and perspectives are finally detailed in section VI. 


\section{SOFT ACTUATOR PRESENTATION}

\section{A. Actuator design}

A Wren mechanism is a type of parallel mechanism [20]. Its parallel structure is of interest to control the chamber stiffness, and large variation of kinematics are possible by adjusting its geometry [22]. A Wren mechanism can be designed as a rigid-body mechanism [23] or a compliant structure [24]. The soft actuator we consider is based on a compliant Wren mechanism using an elastic material, integrated in a chamber made out of a soft material (Fig. 1). In the following, MMAM is considered with Polyjet process (Stratasys, USA). The elastic and soft materials are VeroWhite and TangoBlackPlus materials, following their commercial denominations. The number of legs $n$ of the Wren mechanism is considered as a design variable. The legs are characterized by the angle $\theta_{0}$ and their section $w_{\mathrm{vw}} \times t_{\mathrm{vw}}$. The chamber is of length $L_{0}$, radius $R_{0}$ with a thickness $t_{0}$.

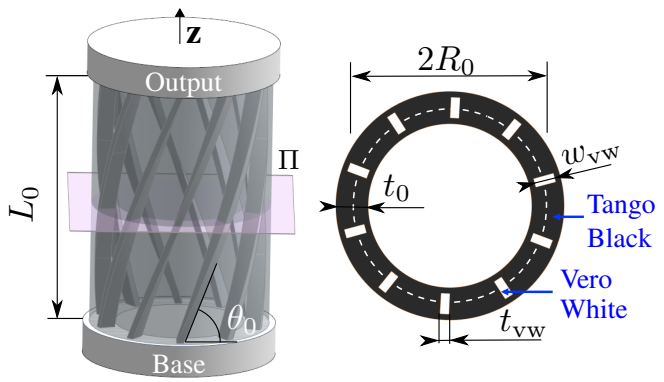

Fig. 1: Structure of the soft actuator: CAD view (left), section in the plane $\pi$ (right).

\section{B. Wren mechanism}

Actuator pressurization can create deformations of top, bottom and lateral surfaces. Top and bottom surfaces of the chamber are chosen thick enough to neglect their deformations. The bottom surface is considered as the base and the top surface as the output of the actuator (Fig. 1). As the lateral surface is mainly composed of a soft material, its deformation will be assessed in next section. The interest of the Wren mechanism for reinforcement however appears when expressing the motion of top surface under pressure.

To estimate the output motion, one can consider the equivalent compliant structure obtained after unwrapping the Wren mechanism to make it planar (Fig. 2a). Such situation corresponds to $R_{0} \rightarrow \infty$. It is then a set of parallel compliant beams, inclined at an angle $\theta_{0}$, connecting the base and output elements. Applying a rigid-body rotation of angle $\theta_{0}$ to the whole structure, we obtain as represented in Fig. $2 \mathrm{~b}$ a compliant stage composed of 2 parallel beams of same length but of shifted axial positions. Such compliant structure is from a kinematic point of view equivalent to a conventional parallel-spring stage with beams at same height [25]. In particular, it is known that the output element exhibits a parabolic path with a horizontal displacement $f$ (Fig. 2b)

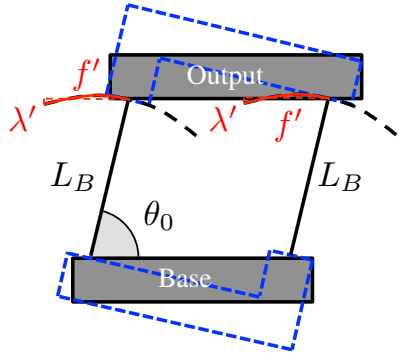

(a) Unwrapped Wren mechanism composed of 2 legs

Fig. 2: Geometrical transformations of Wren mechanism for its kinematic analysis

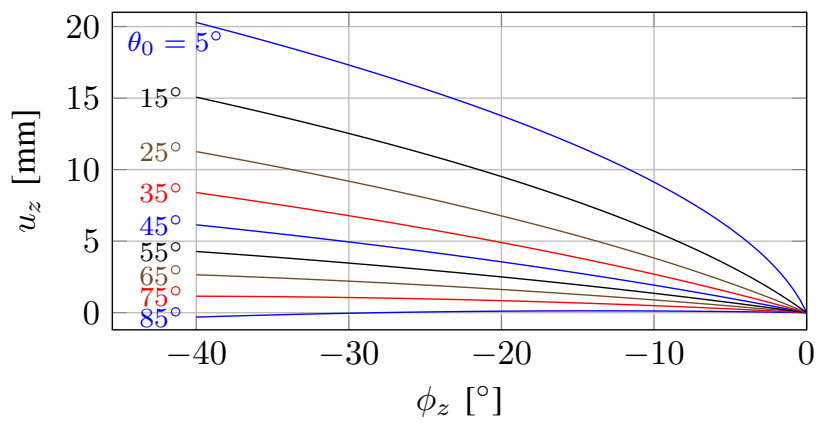

Fig. 3: Output motion of Wren mechanism as a function of the angle $\theta_{0}$ for $L=40 \mathrm{~mm}$ and $R=12 \mathrm{~mm}$.

and a vertical displacement $\lambda$ well approximated [25] by

$$
\lambda=-\frac{3 f^{2}}{5 L_{B}} .
$$

Since the Wren mechanism was initially transformed by a rotation $\theta_{0}$, the displacement $f^{\prime}$ (Fig. 2a) is

$$
f^{\prime}=f \sin \left(\theta_{0}\right)+\lambda \cos \left(\theta_{0}\right) .
$$

The displacement $f^{\prime}$ is linked to the output rotation $\phi_{z}$ with $f^{\prime}=R_{0} \phi_{z}$ so the axial displacement $u_{z}$ of the output element is

$$
u_{z}=\lambda^{\prime}=\lambda \sin \left(\theta_{0}\right)-f \cos \left(\theta_{0}\right) .
$$

From equations (3), (2) and (1), we have finally two possible values for the output axial displacement

$$
\begin{aligned}
u_{z}= & \frac{6 R_{0} \phi_{z} \sin \left(\theta_{0}\right) \cos \left(\theta_{0}\right)-5 L_{B} \sin \left(\theta_{0}\right)}{6 \cos \left(\theta_{0}\right)^{2}} \\
& \pm \frac{\sqrt{5} L_{B}}{6 \cos \left(\theta_{0}\right)^{2}} \sqrt{\frac{5 L_{B} \sin \left(\theta_{0}\right)^{2}-12 R_{0} \phi_{z} \cos \left(\theta_{0}\right)}{L_{B}}}
\end{aligned}
$$

In Fig. 3 the output rotation $\phi_{z}$ and translation $u_{z}$ are represented as a function of the angle $\theta_{0}$. With the use of Wren mechanism, it is possible to control the nature of the output motion. When $\theta_{0}$ is increased to go toward the limit value of $90^{\circ}$, the tangent in $\phi_{z}=0^{\circ}$ tends to become horizontal. This means the output elements shows nearly a pure rotation. When $\theta_{0}$ is close to $0^{\circ}$, the mechanism conversely exhibits a pure translation. 


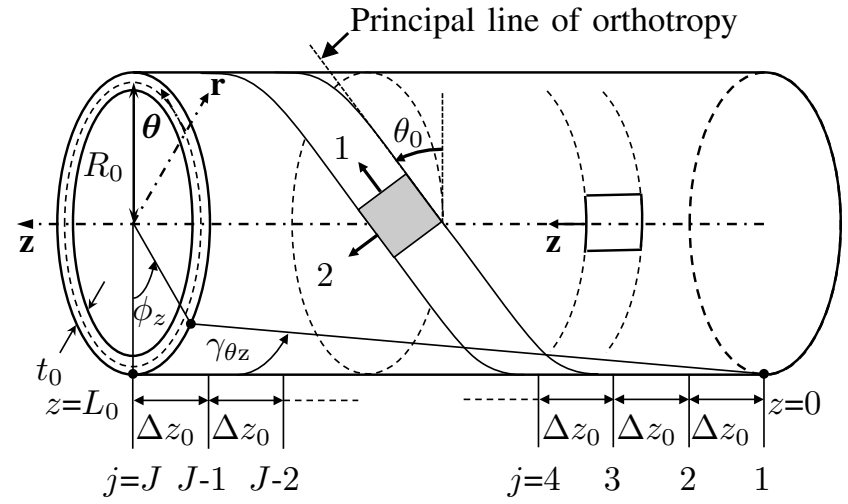

Fig. 4: Homogeneous orthotropic model, adapted from [18].

\section{ACtUATOR MODELING}

\section{A. From composite structure to homogeneous model}

The previous kinematic model does not consider the effect of internal pressure on the sides of the actuator. Initial FEA showed us that the effect of pressure on the chamber sides is indeed significant for the actuator output motions. To model this, we choose to consider the homogeneous orthotropic model of Fig. 4 as a representation of the actuator chamber. We thus assume that the size of the elastic elements in VeroWhite can be considered small with respect to the size of the chamber in TangoBlackPlus material. It may not be well verified after design investigation, so we propose to take into account such model discrepancy by model calibration, as it was successfully performed previously in the context of parallel mechanism kinematic calibration [26].

The homogeneous material of the chamber is then characterized by elastic moduli $E$ and $E^{\prime}$ in the first and second principal directions of the material, denoted 1 and 2 on Fig. 4, the Poisson's ratio $\nu$ and a tangent modulus $G$. The material properties are obtained by considering the elastic and soft materials are placed in parallel in the structure, so a conventional mixture law as used for composite materials [27] can be used:

$$
\begin{array}{ll}
E=c_{\mathrm{vw}} E_{\mathrm{vw}}+c_{\mathrm{tb}} E_{\mathrm{tb}} & \nu=c_{\mathrm{vw}} \nu_{\mathrm{vw}}+c_{\mathrm{tb}} \nu_{\mathrm{tb}} \\
\frac{1}{E^{\prime}}=\frac{c_{\mathrm{vw}}}{E_{\mathrm{vw}}}+\frac{c_{\mathrm{tb}}}{E_{\mathrm{tb}}} & \frac{1}{G}=\frac{c_{\mathrm{vw}}}{G_{\mathrm{vw}}}+\frac{c_{\mathrm{tb}}}{G_{\mathrm{tb}}}
\end{array}
$$

with $E_{\mathrm{vw}}$ and $E_{\mathrm{tb}}$ the Young's modulus of respectively the VeroWhite and TangoBlackPlus materials, $\nu_{\mathrm{vw}}$ and $\nu_{\mathrm{tb}}$ their Poisson's ratio, $G_{\mathrm{vw}}$ and $G_{\mathrm{tb}}$ their tangent modulus, and $c_{\mathrm{vw}}$, $c_{\mathrm{tb}}$ the surface area ratios.

\section{B. Model Derivation}

Orgill and Wilson [18] developed a model for orthotropic cylindrical shells under pressure based on finite differences. The geometry is updated for each load increment to deal with large deformations and displacements. The constitutive relationships are based on a thin-wall assumption, with small radial stress in the chamber. We consider this assumption as valid in our context and derive our actuator model with the same finite-difference numerical scheme. Model validity will be discussed after calibration.
Let $P$ be the pressure in the chamber, $R$ the radius of the chamber, which is dependent on the axial position $z$. The chamber is fully clamped at the bottom $(z=0)$ and the top surface is considered rigid so there is no radial displacement at $z=L$. Following the principle of a finite-difference method, the displacements, stresses and geometrical parameters are considered as functions of the axial position, with a discretization of the chamber length in $(J-1)$ increments $\Delta z$, and the pressure is applied in $I$ steps of pressure, denoted $\Delta P$. According to [18], a pressure increment $\Delta P$ then creates the following mechanical stresses in the chamber wall:

$$
\left\{\begin{aligned}
\Delta \sigma_{\theta \theta i j} & =\frac{\Delta P \cdot R_{(i-1) j}}{t_{(i-1) j}}\left(1-\frac{R_{(i-1) j}}{2 \rho_{(i-1) j}}\right) \\
\Delta \sigma_{z z i j} & =\frac{\Delta P \cdot R_{(i-1) j}}{2 t_{(i-1) j}} \cos ^{2}\left(\alpha_{(i-1) j}\right) \\
\Delta \sigma_{\theta z i j} & =\Delta \sigma_{r r i j}=0
\end{aligned}\right.
$$

with $\Delta \sigma_{\theta \theta i j}, \Delta \sigma_{z z i j}$ and $\Delta \sigma_{\theta z i j}$ respectively the circumferential, longitudinal and shear stresses for load increment $i \in$ $[1, I]$ at location $j \in[1, J]$. The angle $\alpha$ is defined as the angle between the cylinder wall and the axis $\mathbf{z}$, and $\rho$ is the radius of curvature in the meridional direction. From Eq. (6), the strain increment $\Delta \varepsilon_{i j}=\left[\begin{array}{llll}\Delta \varepsilon_{\theta \theta} & \Delta \varepsilon_{z z} & \Delta \varepsilon_{r r} & \Delta \gamma_{\theta z}\end{array}\right]_{i j}^{T}$ can be calculated as

$$
\Delta \varepsilon_{i j}=\boldsymbol{A}_{(i-1) j} \Delta \boldsymbol{\sigma}_{i j}
$$

with $\Delta \boldsymbol{\sigma}_{i j}=\left[\begin{array}{ll}\Delta \sigma_{\theta \theta} & \Delta \sigma_{z z}\end{array}\right]_{i j}^{T}$ the reduced stress vector increment, and the matrix $\boldsymbol{A}_{(i-1) j}$ described by the following non-zero components, denoting $c=\cos \theta_{i j}$ and $s=\sin \theta_{i j}$ :

$$
\left\{\begin{array}{l}
a_{11_{i j}}=\frac{c^{4}}{E}+\left(\frac{1}{G}-\frac{2 \nu}{E}\right) c^{2} s^{2}+\frac{s^{4}}{E^{\prime}} \\
a_{22_{i j}}=\frac{s^{4}}{E}+\left(\frac{1}{G}-\frac{2 \nu}{E}\right) c^{2} s^{2}+\frac{c^{4}}{E^{\prime}} \\
a_{12_{i j}}=a_{21_{i j}}=\left(\frac{1}{E}+\frac{1}{E^{\prime}}+\frac{2 \nu}{E}-\frac{1}{G}\right) c^{2} s^{2}-\frac{\nu}{E} \\
a_{31_{i j}}=a_{32_{i j}}=-\nu / E \\
a_{41_{i j}}=\left[\frac{2 c^{2}}{E}-\frac{2 s^{2}}{E^{\prime}}+\left(\frac{2 \nu}{E}-\frac{1}{G}\right)\left(c^{2}-s^{2}\right)\right] c s \\
a_{42_{i j}}=\left[\frac{2 s^{2}}{E}-\frac{2 c^{2}}{E^{\prime}}-\left(\frac{2 \nu}{E}-\frac{1}{G}\right)\left(c^{2}-s^{2}\right)\right] c s
\end{array}\right.
$$

The increments of radial displacement $\Delta u_{r i j}$, longitudinal displacement $\Delta u_{z i j}$ and circumferential displacement $\Delta u_{\theta i j}$ can then be computed with finite differences:

$$
\left\{\begin{array}{l}
\Delta u_{r i j}=R_{(i-1) j} \Delta \varepsilon_{\theta \theta i j} \\
\Delta u_{z i j}-\Delta u_{z i(j-1)}=\Delta z_{(i-1)(j-1)} \Delta \varepsilon_{z z i j} \\
\Delta u_{\theta i j}-\Delta u_{\theta i(j-1)}=\Delta z_{(i-1)(j-1)} \Delta \gamma_{\theta z i j}
\end{array}\right.
$$

The boundary conditions can be written as $\Delta u_{r i 1}=$ $\Delta u_{z i 1}=\Delta u_{\theta i 1}=0$ and $\Delta u_{r i J}=0$ in (9). Finally, the geometrical parameters and the rotation $\phi_{z i j}$ can be updated for the $i^{t h}$ increment of load, with $u_{x i j}=u_{x(i-1) j}+\Delta u_{x i j}$ :

$$
\left\{\begin{aligned}
R_{i j} & =R_{0} \prod_{k=1}^{i}\left(1+\Delta \varepsilon_{\theta \theta k j}\right) \\
t_{i j} & =t_{0} \prod_{k=1}^{i}\left(1+\Delta \varepsilon_{r r k j}\right) \\
\Delta z_{i j} & =\Delta z_{0}+u_{z i(j(j+1)}-u_{z i j} \\
\theta_{i j} & =\tan ^{-1}\left(\frac{1+\Delta \varepsilon_{z z i j}}{1+\Delta \varepsilon_{\theta \theta i j}} \frac{1}{\cot \theta_{(i-1) j}+\Delta \gamma_{\theta z i j}}\right) \\
\alpha_{i j} & =\tan ^{-1}\left(\frac{\partial u_{r i j}}{\partial z}\right) \\
\rho_{i j} & =\left|\frac{\left(1+\tan ^{2} \alpha_{i j}\right)^{3 / 2}}{\partial^{2} u_{r i j} / \partial z^{2}}\right| \\
\phi_{z i j} & =\phi_{z(i-1) j}+\frac{\Delta u_{\theta i j}}{R_{(i-1) j}}
\end{aligned}\right.
$$




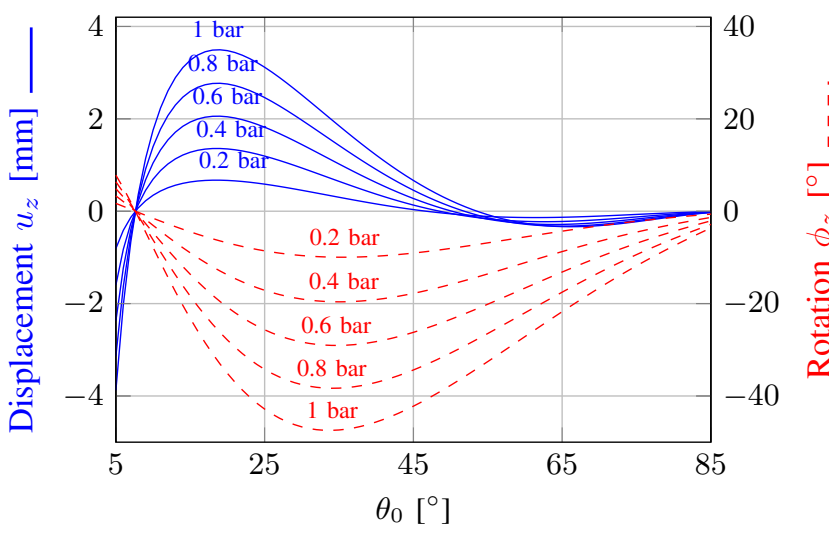

Fig. 5: Prediction of actuator motions

TABLE I: Actuator characteristics for initial prediction of performance

\begin{tabular}{cccccc}
$E_{\mathrm{vw}}$ & $E_{\mathrm{tb}}$ & $G_{\mathrm{vw}}$ & $G_{\mathrm{tb}}$ & $\nu_{\mathrm{vw}}$ & $\nu_{\mathrm{tb}}$ \\
$1830 \mathrm{MPa}$ & $1 \mathrm{MPa}$ & $704 \mathrm{MPa}$ & $0.33 \mathrm{MPa}$ & 0.3 & 0.5 \\
\hline$t_{0}$ & $R_{0}$ & $L_{0}$ & $t_{\mathrm{vw}}$ & $w_{\mathrm{vw}}$ & $n$ \\
$3 \mathrm{~mm}$ & $12 \mathrm{~mm}$ & $40 \mathrm{~mm}$ & $1 \mathrm{~mm}$ & $2.5 \mathrm{~mm}$ & 12
\end{tabular}

with

$$
\left\{\begin{aligned}
\frac{\partial u_{r i j}}{\partial z} & =\frac{u_{r i(j+1)}-u_{r i(j-1)}}{\Delta z_{(i-1) j}+\Delta z_{(i-1)(j-1)}} \\
\frac{\partial^{2} u_{r i j}}{\partial z^{2}} & =\frac{2\left(u_{r i(j+1)}+u_{r i(j-1)}-2 u_{r i j}\right)}{\Delta z_{(i-1) j}^{2}+\Delta z_{(i-1)(j-1)}^{2}}
\end{aligned}\right.
$$

Equations (6)-(11) are computed for each load increment $i$ and each segment $j$ of length $\Delta z_{0}=L_{0} /(J-1)$. Simulation convergence is obtained with $I=200$ and $J=21$. Thanks to the limited complexity of the model, results are obtained in about $0.25 \mathrm{~s}$ using an implementation with Matlab (Mathwork, USA) with an Intel Core i7. It is interesting to note that the maximum computation time observed for one load increment is $25 \mathrm{~ms}$, which would make the model compatible with a use for control of the actuator as well.

\section{Initial Predictions with the Model}

Actuator design will be based on the calibrated model. It is however worth looking at initial model predictions, using the geometrical and material properties indicated in Table I. Such values could indeed be used for actuator prototypes. Translation and rotation of the actuator are reported in Fig. 5. Compared to results of Fig. 3, large values of the angle $\theta_{0}$ allow negative displacements. This is due to the effect of pressure on the chamber sides. According to Fig. 5, the developed device appears of interest to generate in particular pure rotation. Indeed, for $\theta_{0} \approx 55^{\circ}$, translation is close to zero for any pressure. Such observations are made using $a$ priori values for the homogenized material of the chamber. They need thus to be calibrated to take into account the impact of the MMAM process.

\section{Model Calibration}

\section{A. Identification Problem Statement}

In parametric identification, the model parameters $\boldsymbol{\xi}$ are identified by the best fit of experimental measurements $\boldsymbol{m}_{p}$ for different measurement configurations $p$ in order to improve the model accuracy. Each configuration $p$ is described by a set of input parameters $\boldsymbol{\rho}_{p}$. For the calibration of a soft actuator, the parameters are the material properties $\boldsymbol{\xi}=\left[E_{\mathrm{vw}}, E_{\mathrm{tb}}, G_{\mathrm{vw}}, G_{\mathrm{tb}}, \nu_{\mathrm{vw}}, \nu_{\mathrm{tb}}\right]^{T}$ as they do not vary between two different designs of actuators. For actuator evaluation, each measurement configuration is defined by the pressure $P$ and the design parameters so that $\boldsymbol{\rho}_{p}=\left[P^{p}, t_{0}^{p}, R_{0}^{p}, L_{0}^{p}, \theta_{0}^{p}, t_{\mathrm{vw}}^{p}, w_{\mathrm{vw}}^{p}, n^{p}\right]^{T}$. Finally, we define for each pose $p$ the measured outputs as the output displacement $u_{z}$ and rotation $\phi_{z}: \boldsymbol{m}_{p}=\left[u_{z}^{p}, \phi_{z}^{p}\right]^{T}$.

During a conventional identification process like robot calibration, the vector $\boldsymbol{\rho}_{p}$ defines the system workspace [28]. Here, the vector $\rho_{p}$ defines the design workspace of the actuator. However, the same equations arise for calibration in the two situations. It is then of interest to consider conditions on measurement accuracy and measurement workspace as developed in [19] to control the quality of calibration and the improvement of model prediction by experiments. In other words, considering the approach we previously developed in [19], we can ensure the quality of calibration and more precisely the identifiability of the parameters $\boldsymbol{\xi}$ considering the main sources of inaccuracies, here related to the instrumentation and the manufacturing process as well. The methodology is developed in next section.

\section{B. Process \& Experiment Impact}

Two models are needed to develop the conditions we need to verify for the calibration [19]. The first one is the kinematic model of section III that links the input parameters $\boldsymbol{\rho}_{p}$, the model parameters $\boldsymbol{\xi}$ and the outputs $\boldsymbol{x}_{p}=\left[u_{z}^{p}, \phi_{z}^{p}\right]^{T}$ :

$$
\boldsymbol{f}\left(\boldsymbol{\rho}_{p}, \boldsymbol{\xi}, \boldsymbol{x}_{\boldsymbol{p}}\right)=0
$$

The second model is the identification function, that links the input parameters $\boldsymbol{\rho}_{p}$, the model parameters $\boldsymbol{\xi}$ and the measurements $\boldsymbol{m}_{p}$ of pose $p$ :

$$
\boldsymbol{g}\left(\boldsymbol{\rho}_{p}, \boldsymbol{\xi}, \boldsymbol{m}_{p}\right)=0
$$

Since we choose to directly measure the outputs $u_{z}^{p}$ and $\phi_{z}^{p}$, the functions $\boldsymbol{f}$ and $\boldsymbol{g}$ are identical. Their differentiation with respect to the parameters yields to two conditions of calibration, which detailed development is provided in [19], [29]. With $\operatorname{abs}(\bullet)$ the matrix obtained by computing the absolute value of all the components of a matrix, one has

$$
\begin{aligned}
& \forall \boldsymbol{\rho}_{p} \in \mathcal{W}, \quad \operatorname{abs}\left(\boldsymbol{J}_{\xi}^{p}\right) \Delta \boldsymbol{\xi} \leq \Delta \boldsymbol{x}_{f}-\operatorname{abs}\left(\boldsymbol{J}_{\rho}^{p}\right) \Delta \boldsymbol{\rho}^{\boldsymbol{p}} \\
& \forall p=1 . . N_{p}, \quad \Delta \boldsymbol{m}+\operatorname{abs}\left(\boldsymbol{G}_{\rho}^{p}\right) \Delta \boldsymbol{\rho}^{p}<\operatorname{abs}\left(\boldsymbol{G}_{\xi}^{p}\right) \Delta \boldsymbol{\xi}
\end{aligned}
$$

with

- $\mathcal{W}$ the design workspace

- $\Delta \boldsymbol{\rho}^{p}$ the vector of design parameter inaccuracies

- $\Delta \boldsymbol{m}$ the vector of measurement inaccuracies

- $\Delta \boldsymbol{x}_{f}$ the vector of the desired accuracy over $\mathcal{W}$

- $\Delta \boldsymbol{\xi}$ the vector of model parameter accuracies

- $\mathbf{J}_{\xi}^{p}=\boldsymbol{G}_{\xi}^{p}$ respectively the Jacobian matrices of $\boldsymbol{f}$ and $\boldsymbol{g}$ with respect to $\boldsymbol{\xi}$, depending on the pose $p$. 
- $\mathbf{J}_{\rho}^{p}=\boldsymbol{G}_{\rho}^{p}$ respectively the Jacobian matrices of $\boldsymbol{f}$ and $\boldsymbol{g}$ with respect to $\boldsymbol{\rho}$, depending on the pose $p$.

In order to ensure that the conditions (14) and (15) are verified, we need first to define the design workspace when using MAMM process. Our goal is to build a rotary soft actuator, so we set $\theta_{0} \in[40 ; 85]^{\circ}$ in order to be centered around the area of limited translations represented in Fig. 5. Additionally, our prime interest is also to use the actuator in medical context. Compactness is then a key property. We then choose to set $R_{0}=12 \mathrm{~mm}$ and $L_{0}=40 \mathrm{~mm}$ to keep the device small, with a size that is comparable to the needle driver developed in [2]. The other design parameters of $\rho^{p}$ can be freely chosen in the design workspace of Table II. The minimum values of $t_{0}, t_{\mathrm{vw}}, w_{\mathrm{vw}}$ and $n$ are chosen by preliminary evaluation using FEA in order to ensure the component resistance when applying a pressure of 1 bar.

The value of the design parameter inaccuracies $\Delta \rho^{p}$ is defined by taking into account the experimental set-up for actuator evaluation and the MMAM process performance. The pressure $P$ is measured with a pressure sensor (Festo SPTWP6R-G14-VD-M12), which accuracy is equal to $\Delta P=$ 0.06 bar. The MMAM system used for evaluation (Connex 350 , Stratasys, USA) has a printing accuracy measured equal to $\Delta d=85 \mu \mathrm{m}$. The number of legs $n$ is perfectly known, so $\Delta n=0$. One has then with $\Delta \theta_{0}^{p}=\tan ^{-1}\left(\Delta d \sin \theta_{0}^{p} / L_{0}\right)$,

$$
\Delta \boldsymbol{\rho}^{p}=\left[\Delta P, \Delta d, \Delta d, \Delta d, \Delta \theta_{0}^{p}, \Delta d, \Delta d, 0\right]^{T}
$$

Experiments are performed using a laser-based displacement sensor (OptoNCDT 2300-20, Micro-Epsilon) for the measurement of actuator translation. Sensor accuracy is estimated equal to $\Delta u_{z}=20 \mu \mathrm{m}$. Actuator rotation is measured by using using a vision marker located on the top surface. The accuracy is estimated equal to $\Delta \phi_{z}=0.5^{\circ}$. Thus the vector of measurement inaccuracies $\Delta \boldsymbol{m}$ can be written as

$$
\Delta \boldsymbol{m}=\left[\Delta u_{z}, \Delta \phi_{z}\right]^{T}
$$

Acceptable inaccuracies in terms of actuator translation and rotation are related to our final medical application. As we desire to obtain a rotary actuator, we choose to favor the accuracy in translation compared to the one in rotation, and $\Delta \boldsymbol{x}_{f}$ is expressed as

$$
\Delta \boldsymbol{x}_{f}=\left[0.3 \mathrm{~mm}, 5^{\circ}\right]^{T}
$$

Only the vector of model parameter accuracies $\Delta \xi$ is not determined in the conditions (14) and (15). We rather use Eq. (14) to estimate $\Delta \boldsymbol{\xi}$ since the entire design workspace $\mathcal{W}$ is then considered and it may thus be more restrictive. The vector condition (14) yields to 2 scalar inequalities while vector $\Delta \boldsymbol{\xi}$ has 6 components. We set $\Delta E$ and $\Delta \nu$ as the inaccuracies of the elastic modulus $E_{\mathrm{vw}}$ and the Poisson's ratio $\nu_{\mathrm{vw}}$ and from this we derive the inaccuracies for $E_{\mathrm{tb}}$, $G_{\mathrm{vw}}, G_{\mathrm{tb}}, \nu_{\mathrm{tb}}$ by considering the order of magnitude of

TABLE II: Definition of the initial design workspace $\mathcal{W}$.

\begin{tabular}{cccccc}
$P$ & $t_{0}$ & $\theta_{0}$ & $t_{\mathrm{vw}}$ & $w_{\mathrm{vw}}$ & $n$ \\
\hline$[0 ; 1] \mathrm{bar}$ & {$[3 ; 4] \mathrm{mm}$} & {$[40 ; 85]^{\circ}$} & {$[1 ; 2] \mathrm{mm}$} & {$[2.5 ; 5] \mathrm{mm}$} & {$[10 ; 24]$}
\end{tabular}
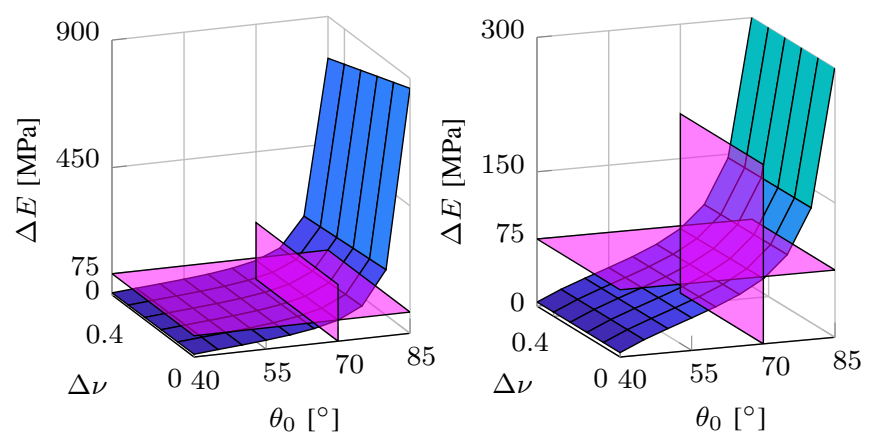

Fig. 6: $\Delta E$ as a function of $\theta_{0}$ and $\Delta \nu$ for the first (left) and second (right) components of Eq. (14).

each parameter. For example, since $G_{\mathrm{vw}} \approx \frac{E_{\mathrm{vw}}}{3}$, we can set $\Delta G_{\mathrm{vw}}=\frac{\Delta E}{3}$. We then obtain

$$
\Delta \boldsymbol{\xi}=\left[\Delta E, \frac{\Delta E}{1000}, \frac{\Delta E}{3}, \frac{\Delta E}{3000}, \Delta \nu, \Delta \nu\right]^{T}
$$

Thus, condition (14) yields 2 inequalities in the two unknowns $\Delta E$ and $\Delta \nu$ over the design workspace $\mathcal{W}$. Computing condition (14) over $\mathcal{W}$ after a discretization, the worst conditions on $\Delta E$ and $\Delta \nu$ are identified to be encountered for $P=1 \mathrm{bar}, t_{0}=3 \mathrm{~mm}, t_{\mathrm{vw}}=1 \mathrm{~mm}, w_{\mathrm{vw}}=2.5 \mathrm{~mm}$ and $n=10$. The condition (14) is represented graphically in Fig. 6 by considering the equality between the two terms in each of the two equations. Inequalities are verified for points below the plotted surfaces. Fig. 6 shows that $\Delta E$ is almost equal to 0 for $\theta_{0} \approx 40^{\circ}$. Such a value of $\Delta E$ is not acceptable since it will then not be possible to satisfy the condition (15) in presence of measurement noises. We therefore reduce the workspace $\mathcal{W}$ to $\theta_{0} \in[70 ; 85]^{\circ}$ in order to set $\Delta \nu=0.1$ and $\Delta E=75 \mathrm{MPa}$.

Condition (15) has to be verified only for the $N_{p}$ calibration poses. The singular values of the identification Jacobian matrices $\boldsymbol{G}_{\xi}^{p}$ have been computed after proper scaling [29]. It appears that only $E_{\mathrm{tb}}$ and $G_{\mathrm{tb}}$ are identifiable among the model parameters $\boldsymbol{\xi}$. Since we have two measurements $u_{z}^{p}$ and $\phi_{z}^{p}$ per pose $p$, one calibration pose can be sufficient. Following [30], this pose is optimized with respect to the observability index $O_{1}$ as defined in [31]. It corresponds to the product of the singular values of the identification matrix $\boldsymbol{G}_{\xi}$. We observed that the actuator designated as $\mathrm{C} 1$ in Table III is then the most interesting with the maximal pressure $P=1$ bar. This is logical as the model parameters $t_{0}, t_{\mathrm{vw}}, w_{\mathrm{vw}}$ and $n$ are at their minimum values, and the pressure at its maximal value, so the device has a low stiffness and exhibits important displacements. Measurement noise has then less impact. In addition, Fig. 5 indicates that the largest displacements when $\theta_{0} \in\left[70^{\circ} ; 85^{\circ}\right]$ are obtained for $\theta_{0}=70^{\circ}$.

For $\mathrm{C} 1$, the condition (15) implies $P>0.5$ bar for the calibration poses. For safety reasons, we also consider two other actuators for calibration, denoted C2 and C3 in Table III, with a larger number of legs to make sure one resists to the pressure. All measurements that respect condition (15) 
TABLE III: Calibration and verification poses. Condition (15) does not concern the verification poses.

\begin{tabular}{c|ccccc|c} 
Actuator & $\begin{array}{c}t_{0} \\
(\mathrm{~mm})\end{array}$ & $\begin{array}{c}\theta_{0} \\
\left({ }^{\circ}\right)\end{array}$ & $\begin{array}{c}t_{\mathrm{vw}} \\
(\mathrm{mm})\end{array}$ & $\begin{array}{c}w_{\mathrm{vw}} \\
(\mathrm{mm})\end{array}$ & $n$ & Condition (15) \\
\hline $\mathrm{C} 1$ & 3 & 70 & 1 & 2.5 & 10 & $P \geq 0.5 \mathrm{bar}$ \\
$\mathrm{C} 2$ & 3 & 70 & 1 & 2.5 & 18 & $P \geq 0.5 \mathrm{bar}$ \\
$\mathrm{C} 3$ & 3 & 70 & 1 & 2.5 & 24 & $P \geq 0.6 \mathrm{bar}$ \\
\hline V4 & 3 & 70 & 1 & 2.5 & 14 & N/A \\
V5 & 3 & 75 & 1 & 2.5 & 14 & N/A \\
V6 & 3 & 79 & 2 & 5 & 12 & N/A \\
V7 & 3 & 73 & 2 & 3.5 & 12 & N/A
\end{tabular}

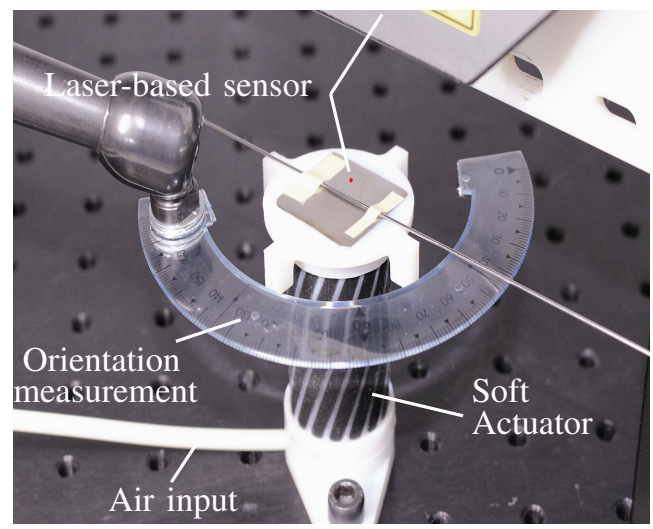

Fig. 7: Experimental set-up.

are used for calibration for these three designs. The other measurements, at low pressure, are used for verification. In order to have also verification poses at high pressure, we consider four other designs, V4 to V7, with different values for the design parameters in $\mathcal{W}$ (see table III).

\section{Experimental process and Results}

The experimental setup is depicted in Fig. 7. The pressure is increased by increments of 0.1 bar and the displacement $u_{z}^{p}$ and rotation $\phi_{z}^{p}$ are measured. All actuators described in Table III resist to a pressure up to 1 bar. Beyond a pressure $P^{\max }$, the actuators do not reach however any steady state in terms of displacements. These pressures and the pressure at breakage $P^{\text {break }}$ are indicated in Table IV. Measurements beyond $P^{\max }$ are not used for the calibration as the absence of steady state makes it difficult for any practical use. The only measurements we use correspond to situations where condition (15) reported in Table III is satisfied. The other measurements for $P<0.5$ bar are used for verification purpose and appear as V1 (resp. V2,V3) for the actuator $\mathrm{C} 1$ (resp. C2, C3) in the results of Fig. 8.

Calibration is performed using a Levenberg-Marquardt algorithm for the optimization, as implemented in Matlab (Mathworks, USA). Fig. 8 shows that errors in translation are satisfactorily decreased for all calibration and verification

TABLE IV: Pressure $P^{\max }$ and $P^{\text {break }}$ for all actuators.

\begin{tabular}{c|ccccccc} 
Actuator & $\mathrm{C} 1$ & $\mathrm{C} 2$ & $\mathrm{C} 3$ & $\mathrm{~V} 4$ & $\mathrm{~V} 5$ & $\mathrm{~V} 6$ & $\mathrm{~V} 7$ \\
\hline$P^{\text {max }}$ (bar) & 0.7 & 1.0 & 1.2 & 0.8 & 0.8 & 1.5 & 1.5 \\
$P^{\text {break }}$ (bar) & 1.7 & 1.4 & 1.5 & 1.7 & 1.3 & $>3.0$ & 2.5
\end{tabular}

TABLE V: Geometrical parameters of the designed rotary actuators and their respective pressure $P^{\max }$.

\begin{tabular}{c|ccccc|c} 
Actuator & $\begin{array}{c}t_{0} \\
(\mathrm{~mm})\end{array}$ & $\begin{array}{c}\theta_{0} \\
0\end{array}$ & $\begin{array}{c}t_{\mathrm{vw}} \\
(\mathrm{mm})\end{array}$ & $\begin{array}{c}w_{\mathrm{vw}} \\
(\mathrm{mm})\end{array}$ & $n$ & $\begin{array}{c}P^{\max } \\
(\text { bar })\end{array}$ \\
\hline $\mathrm{R} 1$ & 4 & 70 & 1.0 & 3.0 & 12 & 0.9 \\
$\mathrm{R} 2$ & 3 & 70 & 1.5 & 3.0 & 16 & 0.6 \\
$\mathrm{R} 3$ & 3 & 70 & 1.0 & 3.0 & 24 & 0.6
\end{tabular}
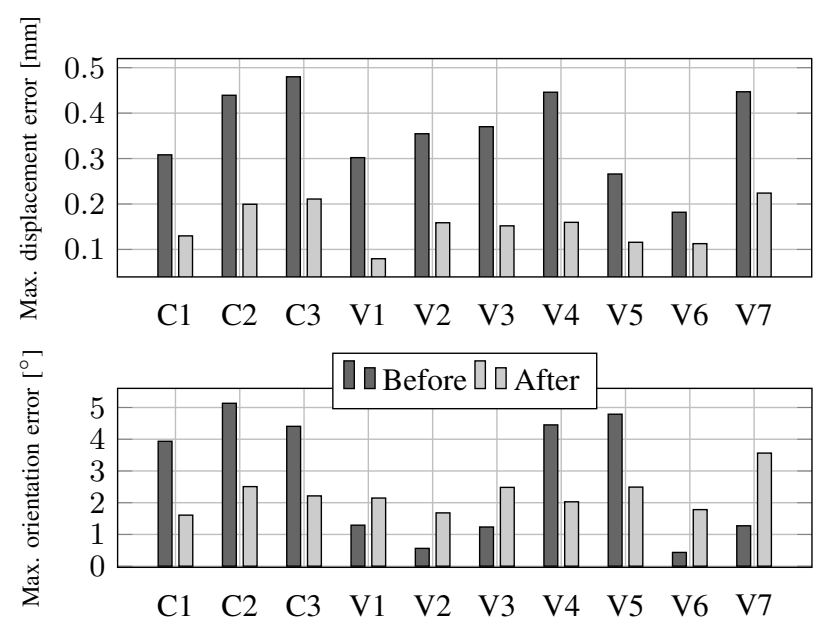

Fig. 8: Calibration errors before and after calibration.

poses. The average reduction is in the order of $58 \%$ for translation and $13 \%$ for rotation on verification poses. Errors in orientation are decreased for the calibration poses, but not for all the verification poses. This is probably linked to our choice of desired accuracy $\Delta \boldsymbol{x}_{f}$ where the accuracy in translation was favoured. However, we can remark that the targeted accuracies are obtained: errors in translation are now always under $0.3 \mathrm{~mm}$ and errors in orientation are always under $5^{\circ}$ after calibration. The identified parameters are $E_{\mathrm{tb}}=1.05 \mathrm{MPa}$ and $G_{\mathrm{tb}}=0.47 \mathrm{MPa}$, which remains somehow close to the a priori values of Table I.

\section{Design BASEd on CALIBRATEd Model}

Rotary soft actuator is built using the identified model to determine the most suitable design parameters in $\mathcal{W}$. We choose to set $\left|\phi_{z}\right|>15^{\circ}$ and $\left|u_{z}\right|<0.1 \mathrm{~mm}$ as an objective for $P \in[0 ; 1]$ bar. The three actuators described in Table V, offering close performances, are produced. Fig. 10 contains the translation and rotation values as obtained from experiments and model predictions using initial and identified parameters. Predictions are plotted for R1, very close results being obtained for R2 and R3. High sensitivity to the model parameters is observed: the direction of translation is opposite to the observed displacement with the initial model. Interesting soft actuator designs are determined thanks to the identification process: the tested geometries offer large rotations $\phi_{z}$ for low translations $u_{z}$, up to $13^{\circ}$ for $0.33 \mathrm{~mm}$ at 0.92 bar for $\mathrm{R} 2$ (see attachment). The model prediction using calibrated values is valid with respect to the targeted accuracy $\Delta \boldsymbol{x}_{f}=\left[0.3 \mathrm{~mm}, 5^{\circ}\right]^{T}$, while it is not true with initial values, which confirms the interest of identification. 


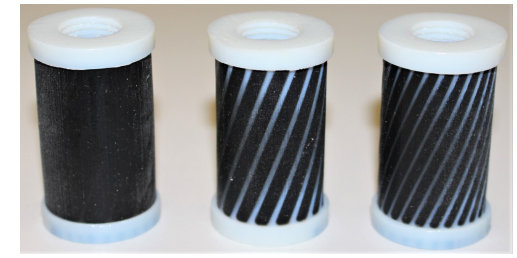

Fig. 9: Actuators R1, R2 and R3 (from left to right)

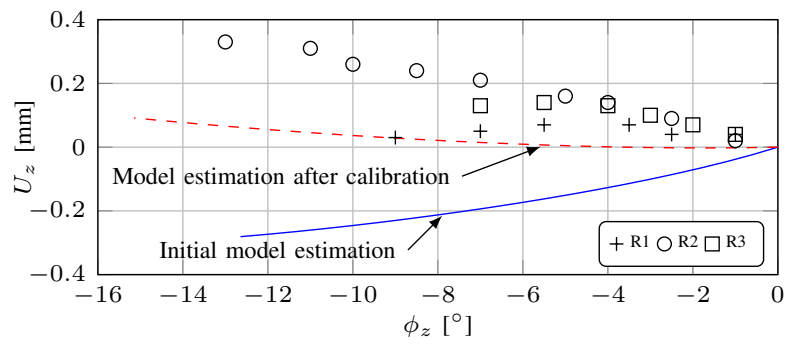

Fig. 10: Experimental results for R1, R2 and R3.

Actuators R1 and R3 do not reach steady state for $P>$ 0.6 bar. Visco-elastic effects seem to govern the actuator behavior, as observed during some calibration tests. This reveals the potential need for non-linear modeling of chamber material. The model we selected can indeed take this into account, modifying the stress-strain relationship in Eq. (8). This will be considered as further development.

\section{CONCLUSION}

In this paper, calibration techniques are associated to a finite-difference model of orthotropic pressurized chamber to predict performance of soft actuator. The approach ensures model identifiability, taking into account performance of experimental characterization and also the process specifications. Its interest was observed considering a soft actuator based on compliant Wren mechanism. In addition, the evaluated design appears promising in building a soft rotary actuator, in particular for the use in medical context. Application of the proposed calibration process to other types of soft actuators will now be considered, as well as the development of the model with non-linear properties material. Integration of Wren-based soft actuator in a robotic device for surgical tool manipulation will also be investigated.

\section{REFERENCES}

[1] D. Rus and M.T. Tolley. Design, fabrication and control of soft robots. Nature, 521(7553):467-475, 2015.

[2] A. Pfeil, L. Barbé, B. Wach, A. Bruyas, F. Geiskopf, M. Nierenberger, and P. Renaud. A 3D-printed needle driver based on auxetic structure and inchworm kinematics. In ASME Int. Des. Eng. Tech. Conf., 2018.

[3] R. Gaylord. Fluid actuated motor system and stroking device, 1958.

[4] F. Daerden and D. Lefeber. Pneumatic artificial muscles: Actuators for robotics and automation. Eur. J. of Mech. and Env. Eng., 47(1):11-21, 2002.

[5] D. Villegas, M. Van Damme, B. Vanderborght, P. Beyl, and D. Lefeber. Third generation pleated pneumatic artificial muscles for robotic applications: Development and comparison with McKibben muscle. Adv. Rob., 26(11):1205-1227, 2012.

[6] L. Pez, G. Agarwal, and J. Paik. Design and analysis of a soft pneumatic actuator with origami shell reinforcement. Soft Rob., 3(3):109-119, 2016.
[7] D. B. Comber, J. E. Slightam, V. R. Gervasi, J. S. Neimat, and E. J. Barth. Design, Additive Manufacture, and Control of a Pneumatic MR-Compatible Needle Driver. IEEE Trans. on Rob., 32(1):138-149, 2016.

[8] D. Drotman, S. Jadhav, M. Karimi, P. deZonia, and M.T. Tolley. 3D printed soft actuators for a legged robot capable of navigating unstructured terrain. In IEEE Int. Conf. on Rob. and Autom., pages 5532-5538, 2017.

[9] N. Bartlett, M.T. Tolley, Overvelde J., J. Weaver, B. Mosadegh, K. Bertoldi, G. Whitesides, and R. Wood. Soft robotics. A 3D-printed, functionally graded soft robot powered by combustion. Science, 349(6244):161-165, 2015.

[10] A. Bruyas, F. Geiskopf, and P. Renaud. Design and Modeling of a Large Amplitude Compliant Revolute Joint: The Helical Shape Compliant Joint. ASME J. of Mech. Design, 137(8):085003, 2015.

[11] S. Davis, N. Tsagarakis, J. Canderle, and D. Caldwell. Enhanced modelling and performance in braided pneumatic muscle actuators. Int. J. of Rob. Research, 22(3-4):213-227, 2003.

[12] P. Polygerinos, Z. Wang, J. Overvelde, K. Galloway, R. Wood, and C. Walsh. Modeling of soft fiber-reinforced bending actuators. IEEE Trans. on Rob., 31(3):778-789, 2015.

[13] J. Takosoglu, P. Laski, S. Blasiak, and G. Bracha. Determining the static characteristics of pneumatic muscles. Meas. and Control, 49(2):62-71, 2016.

[14] P. Nguyen, S. Sridar, W. Zhang, and P. Polygerinos. Design and control of a 3-chambered fiber reinforced soft actuator with off-theshelf stretch sensors. Int. J. of Int. Rob. and Applications, 1:342-351, 2017.

[15] J. Bishop-Moser and S. Kota. Design and modeling of generalized fiber-reinforced pneumatic soft actuators. IEEE Trans. on Rob. 31(3):536-545, 2015

[16] B. Zhu, C. Rahn, and C. Bakis. Actuation of fluidic flexible matrix composites in structural media. J. of Int. Mat. Syst. and Structures, 23(3):269-278, 2011.

[17] J.F. Wilson and G. Orgill. Linear analysis of uniformly stresses orthotropic cylindrical shells. ASME J. of Applied Mech., 53(2):249256, 1986.

[18] G. Orgill and J.F. Wilson. Finite deformations of nonlinear, orthotropic cylindrical shells. ASME J. of Applied Mech., 53(2):257-265, 1986.

[19] T. Gayral and D. Daney. A sufficient condition for parameter identifiability in robotic calibration. In Comp. Kinematics, pages 131138. Springer, 2014.

[20] K. Wohlhart. Kinematotropic Linkages, pages 359-368. Springer Netherlands, Dordrecht, 1996.

[21] N. Yee and G. Coghill. Modelling of a novel rotary pneumatic muscle. In Australasian Conf. on Rob. and Autom., pages 186-190, 2002.

[22] K. Zhang and J. S. Dai. Reconfiguration analysis of Wren platform and its kinematic variants based on reciprocal screw systems. In IFToMM World Cong. in Mech. and Mach. Science, 2015.

[23] R. Vertechy and V. Parenti-Castelli. Static and stiffness analyses of a class of over-constrained parallel manipulators with legs of Type US and UPS. In IEEE Int. Conf. on Rob. and Autom., pages 561-567, 2007.

[24] R. Wang, X. Zhou, Z. Zhu, and Q. Liu. Compliant linear-rotation motion transduction element based on novel spatial helical flexure hinge. Mech. and Mach. Theory, 92:330-337, 2015.

[25] L. Rubbert, R. Bitterli, N. Ferrier, S. Fifanski, I. Vardi, and S. Henein. Isotropic springs based on parallel flexure stages. Prec. Eng., 43:132145, 2016.

[26] T. Gayral, D. Daney, and M. Bernot. Model discrepancy in robotic calibration: Its influence on the experimental parameter identification of a parallel space telescope. In IEEE/RSJ Int. Conf. on Int. Rob. and Systems, pages 5803-5808, 2013.

[27] H. Altenbach, J. Altenbach, and W. Kissing. Mechanics of composite structural elements. Springer, 2004.

[28] B. Mooring, M. Driels, and Z. Roth. Fundamentals of Manipulator Calibration. John Wiley \& Sons, Inc., New York, USA, 1991.

[29] T. Gayral. Étalonnage d'un instrument d'observation spatial actif. $\mathrm{PhD}$ thesis, Université Nice Sophia Antipolis, 2013.

[30] D. Daney, Y. Papegay, and B. Madeline. Choosing measurement poses for robot calibration with the local convergence method and Tabu search. Int. J. of Rob. Res., 24(6):501-518, 2005.

[31] Y. Sun and J. M. Hollerbach. Observability index selection for robot calibration. In IEEE Int. Conf. on Rob. and Autom., pages 831-836, Pasadena, CA, 2008. 